This is a self-archived version of an original article. This version may differ from the original in pagination and typographic details.

Author(s): Silaev, M. A.; Bobkova, I. V.; Bobkov, A. M.

Title: Odd triplet superconductivity induced by a moving condensate

Year: 2020

Version: Published version

Copyright: @2020 American Physical Society

Rights: In Copyright

Rights url: http://rightsstatements.org/page/lnC/1.0/?language=en

Please cite the original version:

Silaev, M. A., Bobkova, I. V., \& Bobkov, A. M. (2020). Odd triplet superconductivity induced by a moving condensate. Physical Review B, 102(10), Article 100507.

https://doi.org/10.1103/PhysRevB.102.100507 


\title{
Odd triplet superconductivity induced by a moving condensate
}

\author{
M. A. Silaev, ${ }^{1,2}$ I. V. Bobkova $\oplus_{, 3,2,4}$ and A. M. Bobkov ${ }^{3}$ \\ ${ }^{1}$ University of Jyvaskyla, Department of Physics and Nanoscience Center, P.O. Box 35 (YFL), \\ FI-40014 University of Jyväskylä, Finland \\ ${ }^{2}$ Moscow Institute of Physics and Technology, Dolgoprudny 141700, Russia \\ ${ }^{3}$ Institute of Solid State Physics, Chernogolovka, Moscow Region 142432, Russia \\ ${ }^{4}$ Dubna State University, Dubna 141980, Russia
}

(Received 8 January 2020; revised 9 March 2020; accepted 10 September 2020; published 28 September 2020)

\begin{abstract}
It has been commonly accepted that a magnetic field suppresses superconductivity by inducing the ordered motion of Cooper pairs. We demonstrate that a magnetic field can instead provide a generation of superconducting correlations by inducing the motion of a superconducting condensate. This effect arises in superconductor/ferromagnet heterostructures in the presence of Rashba spin-orbital coupling. We predict the odd-frequency spin-triplet superconducting correlations called the Berezinskii order to be switched on at large distances from the superconductor/ferromagnet interface by the application of a magnetic field. This is shown to result in the unusual behavior of Josephson effect and local density of states in superconductor/ferromagnet structures.
\end{abstract}

\section{DOI: 10.1103/PhysRevB.102.100507}

The phenomenon of superconductivity dwells on the condensation of Cooper pairs each consisting of two electrons with opposite momenta. A magnetic field tends to break Cooper pairs by inducing their center-of-mass motion which makes the momenta of two paired electrons to be not exactly opposite. This seems to be the fundamental mechanism called the orbital depairing effect which exists in any superconducting system and leads to the suppression of superconductivity by the magnetic field [1].

In this Rapid Communication we show that the magnetic field can in fact stimulate Josephson current by generating correlations which are odd in the frequency domain. Our proposal is based on the observation that the combination of exchange field, spin-orbit coupling (SOC), and controllable condensate motion is generically enough for the effective manipulation of the odd-frequency spin-triplet pairing states [2] which have attracted continual interest for several decades [3-27]. We find that the condensate motion generated by the external magnetic field through the Meissner effect adds an additional degree of freedom which can control the generation of odd-frequency superconductivity in currently available experimental setups with SOC [28-34]. In the context of superconductor (S)/ferromagnet (F) hybrid structures [35-44] these correlations are known as long-range triplets (LRTs) because they can penetrate at large distances into the ferromagnetic material $[9,45-60]$. We suggest a mechanism which leads to the stimulation of long-range Josephson current in SFS junctions by the external magnetic field thus opening great perspectives for low-dissipative spintronics [61,62].

Recently the phenomena employing SOC in the SF structures has attracted much attention [30,34,63-86]. The SOC is generated at interfaces due to the broken inversion symmetry and usually has Rashba-type form [87-91] $(\alpha / m)(\hat{\boldsymbol{\sigma}} \times \boldsymbol{p}) \cdot \boldsymbol{n}$ where $\boldsymbol{n}$ is the interface normal, $\hat{\boldsymbol{\sigma}}$ is the vector of spin Pauli matrices, and $\boldsymbol{p}$ and $m$ are the electron momentum and mass. It can be additionally enhanced by adding a thin layer of heavy metal $[90,91]$ as sketched in Fig. 1. Usual values of interfacial Rashba level splitting in metals can be assumed to be small, $v_{F} \alpha / E_{F} \ll 1$, where $v_{F}$ and $E_{F}$ are the Fermi velocity and energy, respectively. Under this condition the modification of spin-singlet pairing by the SOC alone is negligible $[67,73,92-$ 94]. However, even in this case the combined effect of SOC and spin-splitting field shows up in the quite efficient generation of spin-triplet superconducting correlations.

The combination of interfacial SOC and exchange field can induce $p$-wave spin-triplet superconducting correlations [64] which mediate the long-range Josephson effect through clean ferromagnets $[63,95]$. In a diffusive system $p$-wave correlations decay at the mean free path which is much smaller than the thickness of the F layer. At the same time $p$-wave correlations exist near the interface with nonzero SOC. They can be converted to $s$-wave correlations due to the anisotropic Doppler shift of quasiparticle energy levels [96] $\boldsymbol{v}_{F} \cdot \boldsymbol{p}_{s}$ induced by the condensate motion along the interface as shown in Fig. 1(b). This shows up in the generation of LRT pairs which are robust to both disorder and exchange interaction. This effect can be engineered combining usual superconductors such as $\mathrm{Al}$ or $\mathrm{Nb}$ with ferromagnets and $\mathrm{SOC}$ [70-72,75,86,97-99].

Without the supercurrent LRTs are absent in the generic S/F structures such as shown in Fig. 1(a). Here the exchange field $\boldsymbol{h} \| \boldsymbol{z}$ produces only short-range triplets with $S_{z}=0$ shown schematically by the blue arrows which decay at short length of the order $\xi_{F} \sim 1 \mathrm{~nm}$ in usual ferromagnets. We demonstrate that the generation of LRTs with $S_{z}= \pm 1$ shown schematically by red arrows in Fig. 1(b) can be achieved by inducing the superconducting condensate momentum satisfying the condition $\boldsymbol{h} \times\left(\boldsymbol{n} \times \boldsymbol{p}_{s}\right) \neq 0$. This effect is fully 

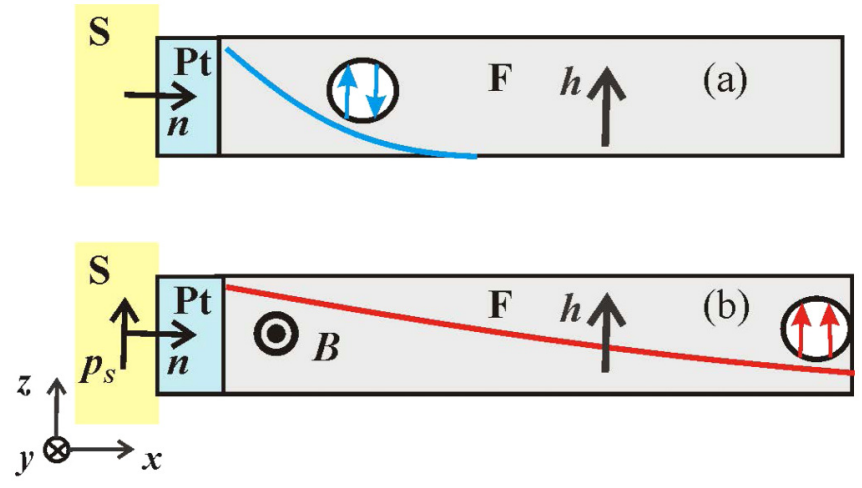

FIG. 1. Schematic picture of the system considered: diffusive superconductor/ferromagnet junction with Rashba SOC at the S/F interface induced by the thin heavy-metal Pt layer. (a) Only shortrange superconducting correlations are present in the absence of a magnetic field. (b) A magnetic field generates condensate momentum through the Meissner effect $\boldsymbol{p}_{s}=-\left(\lambda_{L} / \Phi_{0}\right) \boldsymbol{n} \times \boldsymbol{B}$. The interplay of condensate momentum $\boldsymbol{p}_{s}$, SOC, and exchange field $\boldsymbol{h}$ leads to the generation of a long-range $s$-wave spin-triplet component for $\boldsymbol{B} \neq 0$.

controllable by the external magnetic field $\boldsymbol{B} \perp \boldsymbol{h}$ which induces the supercurrent due to the Meissner effect $\boldsymbol{p}_{s}=$ $-\left(\lambda_{L} / \Phi_{0}\right) \boldsymbol{n} \times \boldsymbol{B}$ where $\lambda_{L}$ is the London penetration length and $\Phi_{0}$ is the magnetic flux quantum.

The qualitative physics of the effect can be described as follows. We need three ingredients to generate LRTs: spin-splitting field $\boldsymbol{h}$, SOC, and supercurrent or condensate momentum $\boldsymbol{p}_{s}$. The role of $\boldsymbol{h} \| \boldsymbol{z}$ is to generate $S_{z}=0$ spintriplet correlations. The role of SOC is to convert them to $S_{z}= \pm 1$ correlations due to the momentum-dependent spin rotation. The general structure of anomalous function describing the pairing in the spin-triplet channel can be parametrized [100] as $\left(\hat{\boldsymbol{\sigma}} \cdot \boldsymbol{d}_{p w}\right)$, where $\hat{\boldsymbol{\sigma}}$ is the vector of Pauli matrices. The p-wave spin vector is $\boldsymbol{d}_{p w}=F_{p w}(\omega) \boldsymbol{h} \times(\boldsymbol{n} \times \boldsymbol{p})$ with the amplitude $F_{p w}(\omega)$ which is an even function of the Matsubara frequency $\omega$.

By the order of magnitude $\left|\boldsymbol{d}_{p w}\right| \sim h v_{F} \alpha / \Delta^{2} \gg v_{F} \alpha / E_{F}$ which in principle is not small. These triplets are even-frequency $\boldsymbol{d}_{p w}(\omega)=\boldsymbol{d}_{p w}(-\omega)$ and odd-parity $p$-wave $\boldsymbol{d}_{p w}(\boldsymbol{p})=-\boldsymbol{d}_{p w}(-\boldsymbol{p})$ correlations. Depending on the relative orientation of the Zeeman field $\boldsymbol{h}$ and Rashba vector $\boldsymbol{n}$ the spin vector field $\boldsymbol{d}_{p w}$ can form different textures in momentum space. In Figs. 2(a) and 2(c) the two characteristic examples of the hedgehog and domain wall are shown, respectively.

The externally induced superflow $\boldsymbol{p}_{s} \neq 0$ induces Doppler shift of the quasiparticle energy levels $[96,101] \boldsymbol{v}_{F} \cdot \boldsymbol{p}_{s}$. It results in the suppression of pairing on one part of the Fermi surface, namely, for electrons with momentum $\boldsymbol{p} \| \boldsymbol{p}_{s}$. In the simplest case of a homogeneous system this leads to the shift of imaginary frequencies so that the amplitude of triplet correlations is given by $F_{p w}\left(\omega-i \boldsymbol{v}_{F} \cdot \boldsymbol{p}_{s}\right) \approx$ $F_{p w}(\omega)-i\left(\boldsymbol{v}_{F} \cdot \boldsymbol{p}_{s}\right) \partial_{\omega} F_{p w}$. This modification of the pairing amplitude results in the additional component of the spin vector $\delta \boldsymbol{d}=-i \partial_{\omega} F_{p w}\left(\boldsymbol{v}_{F} \cdot \boldsymbol{p}_{s}\right) \boldsymbol{h} \times(\boldsymbol{n} \times \boldsymbol{p})$. Quite interesting for us is the $s$-wave component $\boldsymbol{d}_{s w}=\langle\delta \boldsymbol{d}\rangle_{p}$ given by $\boldsymbol{d}_{s w}=(2 / 3 i) E_{F}\left(\partial_{\omega} F_{p w}\right) \boldsymbol{h} \times\left(\boldsymbol{n} \times \boldsymbol{p}_{s}\right)$. The typical amplitude is $\left|\boldsymbol{d}_{s w}\right| \sim\left(p_{s} \xi\right) h v_{F} \alpha / \Delta^{2}$ where $\xi$ is the coherence length.
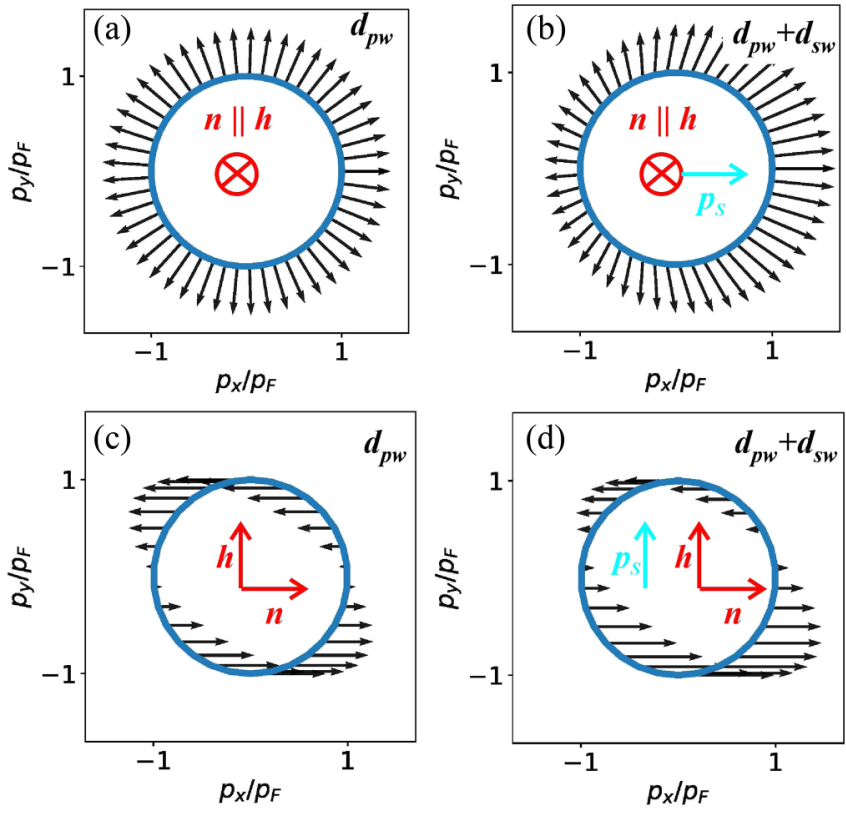

FIG. 2. Textures of the spin-triplet order parameter vector $\boldsymbol{d}$ on the Fermi surface $p_{z}=0$ cross section. Left column: odd-parity state $\boldsymbol{d}_{p w}$ for resting condensate $\boldsymbol{p}_{s}=0$. (a) $\boldsymbol{n} \| \boldsymbol{h}$. (c) $\boldsymbol{n} \perp \boldsymbol{h}$. Right column: mixture of odd- and even-parity states $\boldsymbol{d}_{p w}+\boldsymbol{d}_{s w}$ induced by the moving condensate $\boldsymbol{p}_{s} \neq 0$. (b) $\boldsymbol{h} \| \boldsymbol{n} \perp \boldsymbol{p}_{s}$ and (d) $\boldsymbol{h} \| \boldsymbol{p}_{s} \perp \boldsymbol{n}$.

Examples of spin textures consisting of the even- and oddparity mixtures $\boldsymbol{d}_{p w}+\boldsymbol{d}_{s w}$ are shown in Figs. 2(b) and 2(d). To produce both the $p$-wave and $s$-wave triplets it is crucial to have $h \neq 0$ so that they are qualitatively different from those obtained beyond quasiclassical approximation due to the Edelstein effect $[67,94,95]$.

The described mechanism has the same physical origin as the one reported in Ref. [98], where the gradients of pairing amplitude arising near the edges of S and Pt layers in the planar geometry in combination with Rashba SOC can generate LRTs. In our proposal LRTs are generated within the entire Pt layers thus providing much larger long-range Josephson currents. The other major advantage of our proposal is the full direct magnetic and electric control over the LRT generation because the condensate motion can be induced by applying an external supercurrent or the external magnetic field.

We assume that SOC is small, $v_{F} \alpha / E_{F} \ll 1$, which allows neglecting magnetoelectric effects [102] and spontaneous phase gradients $[65,73,83]$. Simultaneously this assumption allows using the quasiclassical theory describing the system in terms of the quasiclassical propagator $\breve{g}=\breve{g}(\omega, \boldsymbol{p}, \boldsymbol{r})$, which is a $4 \times 4$ matrix in the direct product of particle-hole and spin spaces, and $\omega$ is the imaginary frequency. It is determined by the Eilenberger equation [103]

$$
\left(\boldsymbol{v}_{F} \cdot \hat{\boldsymbol{\partial}}\right) \hat{g}=[\check{\Lambda}, \hat{g}]+\tau_{\text {imp }}^{-1}\left[\langle\hat{g}\rangle_{p}, \hat{g}\right] .
$$

Here $\boldsymbol{v}_{F}=\boldsymbol{p} / m$ is the Fermi velocity, $\hat{\partial} \hat{g}=\nabla \hat{g}-i[\alpha \hat{\mathcal{A}}+$ $\left.\frac{A}{2 \Phi_{0}} \hat{\tau}_{3}, \hat{g}\right]$ is the covariant gradient, and $\hat{\tau}_{i}$ are Pauli matrices in particle-hole space. The quasiclassical equations are supplemented by the normalization condition $\breve{g}^{2}=1$. We denote $\check{\Lambda}=\hat{\tau}_{3}(\omega+i \boldsymbol{h} \cdot \hat{\boldsymbol{\sigma}}-i \hat{\Delta})$, where $\hat{\Delta}=|\Delta| e^{i \hat{\tau}_{3} \chi} \hat{\tau}_{1}$ is the superconducting order parameter, $\boldsymbol{A}$ is the vector potential, and $\hat{\mathcal{A}}$ 
is the general $\mathrm{SU}(2)$ field describing SOC. The last term in Eq. (1) describes impurity scattering with the rate $\tau_{\mathrm{imp}}^{-1}$ and $\langle\cdots\rangle_{p}$ denotes the average over directions of $\boldsymbol{p}$.

First, to elucidate the mechanism behind the generation of $s$-wave spin triplets let us consider solutions of Eq. (1) with spatially homogeneous fields $|\Delta|, \boldsymbol{h}, \hat{\mathcal{A}}$, and the gauge-invariant condensate momentum $\boldsymbol{p}_{s}=\nabla \chi-\boldsymbol{A} / \Phi_{0}$. We consider expansion by small parameters $v_{F} \alpha / \Delta \ll 1$ and $v_{F} p_{s} / \Delta \ll 1$. Let us denote as $\hat{g}_{h}$ the zeroth-order solution of Eq. (1) with $\alpha=0$ and $\boldsymbol{p}_{s}=0$. The first-order correction by SOC is [104]

$$
\hat{g}_{\alpha}=i \alpha \hat{g}_{h}\left[\hat{g}_{h},\left(\boldsymbol{v}_{F} \cdot \hat{\mathcal{A}}\right)\right] /\left(s_{+}+s_{-}+2 \tau_{\mathrm{imp}}^{-1}\right),
$$

where $s_{ \pm}=\sqrt{(\omega \pm i h)^{2}+\Delta^{2}}$. These corrections generate pairing in a $p$-wave even-frequency spin-triplet channel which is known to exist in superfluid ${ }^{3} \mathrm{He}$ and systems with Rashba SOC [92]. For Rashba SOC, $\hat{\mathcal{A}}=\boldsymbol{n} \times \hat{\boldsymbol{\sigma}}$, the anomalous part of Eq. (2) is given by [104] $\left(\hat{\boldsymbol{\sigma}} \cdot \boldsymbol{d}_{p w}\right)$ with the amplitude $F_{p w}=-i \alpha \Delta /\left[m s_{+} s_{-}\left(s_{+}+s_{-}+2 \tau_{\text {imp }}^{-1}\right)\right]$. By the order of magnitude $\hat{g}_{\alpha} \sim h v_{F} \alpha / \Delta^{2}$.

As we discussed above, the condensate momentum adds Doppler shift to the Matsubara frequency in Eq. (1). This results in the $s$-wave spin-triplet correction which in the clean limit is given by $\hat{g}_{\alpha A}=-\left(\boldsymbol{v}_{F} \cdot \boldsymbol{p}_{s}\right) \partial_{\omega} \check{g}_{\alpha}$ which contains the $s$-wave component with the spin structure described by the vector $\boldsymbol{d}_{s w} \propto \boldsymbol{h} \times\left(\boldsymbol{n} \times \boldsymbol{p}_{s}\right)$. By the order of magnitude $\hat{g}_{\alpha A} \propto\left(\xi^{2} \alpha h p_{s} / \Delta\right)$ where $\xi=v_{F} / \Delta$ is the coherence length in the ballistic limit $\tau_{\text {imp }}^{-1}=0$ and $\xi=\sqrt{D / \Delta}$ in the dirty limit $\Delta \tau_{\text {imp }} \ll 1$ where $D$ is the diffusion coefficient.

The mechanism which we have discussed for the minimal model of a homogeneous system works as well for the proximity system where the spin-splitting field is provided by the exchange interaction in the ferromagnet adjacent to the superconductor. The most striking demonstration of the spin-triplet correlations with $\boldsymbol{d}_{s w} \perp \boldsymbol{h}$ can be achieved in a S/F structure with Rashba SOC shown in Fig. 1. Below we show that the generation of LRTs in the ferromagnet can be fully controlled by the external magnetic field. This effect and its experimental manifestations are discussed below.

LRT Josephson effect in SFS junction stimulated by a magnetic field. For the setup in Fig. 1(a) with $\boldsymbol{B}=0$ we have $\boldsymbol{d}_{s w}=0$ and therefore despite the presence of SOC only $S_{z}=0$ Cooper pairs are generated. Such correlations decay in the diffusive ferromagnet at the length scale $\xi_{F}=\sqrt{D / h}$ which is rather short- $\xi_{F} \sim 1 \mathrm{~nm}$ in usual materials. Thus with exponential accuracy the superconductivity is absent at the distances $x \gg \xi_{F}$ from the S/F interface.

At the same time the correlations with $\boldsymbol{d}_{s w} \neq 0$ that appear at $\boldsymbol{B} \neq 0$ have $S_{z}= \pm 1$. Therefore they are LRTs which are robust to the spin depairing and the only correlations that survive at the distances $x \gg \xi_{F}$ from the $\mathrm{S} / \mathrm{F}$ interface in the ferromagnet. In the setup shown in Fig. 1(a), such pairs appear only upon applying the magnetic field $\boldsymbol{B} \perp \boldsymbol{h}$. Hence we claim to find the mechanism of the odd triplet superconductivity generated by the magnetic field. The formation of LRTs has important consequences in the transport properties [54-56,105-107] which can be directly measured using the electrical probes. Below we discuss two of them-the tunnel conductance and Josephson current.
To describe the diffusive system we use a Usadel equation for the $s$ component of the Green's function. It is obtained from the general Eilenberger equation (1) in the limit of large impurity scattering $\tau_{\text {imp }} h \ll 1$. Here we discuss a simplified version of the Usadel theory using the following assumptions. First, we consider tunneling S/Pt interfaces in Fig. 1 so that superconducting correlations are small both in Pt and in F. This allows one to linearize the Usadel equation by assuming $\hat{g}=\operatorname{sgn}(\omega) \hat{\tau}_{3}+\check{f}$ where the anomalous part $\check{f}=\hat{f}_{s}+\hat{\boldsymbol{f}}_{t} \cdot \hat{\boldsymbol{\sigma}}$ has spin-singlet $\hat{f}_{s}$ and spin-triplet $\hat{f}_{t}$ components.

Second, we assume that SOC is localized within Pt layers of the width $d_{s o c} \ll \xi_{N}$, where $\xi_{N}=\sqrt{D / 2 \pi T}$. That is, integrating the Usadel equation through the Pt layer we get the boundary condition at the effective S/F interfaces [104]

$$
\begin{gathered}
n_{x} \nabla_{x} \hat{f}_{s}=\gamma \hat{F}_{b c s}, \\
n_{x} \nabla_{x} \hat{\boldsymbol{f}}_{t}=4 i \tilde{\alpha} \hat{\tau}_{3} \hat{\boldsymbol{f}}_{t} \times\left(\boldsymbol{p}_{s} \times \boldsymbol{n}\right),
\end{gathered}
$$

where $\hat{F}_{b c s}=i \tau_{3} \hat{\Delta} / \sqrt{\omega^{2}+\Delta^{2}}$ and the surface SOC strength $\tilde{\alpha}=\int d x \alpha(x)$ and $\gamma$ is the $\mathrm{S} / \mathrm{F}$ interface transparency $[9,108]$. Note that the boundary condition (4) corresponds to the conversion of $p$-wave to $s$-wave correlations shown in Fig. 2. Indeed, the right-hand side of Eq. (4) is equal to $-i \int d x\left\langle\left(\boldsymbol{p} \cdot \boldsymbol{p}_{s}\right)\left[\hat{\tau}_{3}, \hat{g}_{p w}\right]\right\rangle_{p}$, where $\hat{g}_{p w}$ is the $p$-wave component of $\hat{g}$ and the integration is done over the Pt layer with $\alpha \neq 0$.

Finally, assuming that $p_{s} \xi \ll 1$, we neglect the orbital depairing term $p_{s}^{2} D$ from the Usadel equation. That is, only the linear in $\boldsymbol{p}_{s}$ term described by the boundary condition (4) is retained. Then, in the $\mathrm{F}$ layer where $\alpha=0$ and $\boldsymbol{h} \neq 0$, we obtain the linearized Usadel equations:

$$
\begin{gathered}
\frac{D}{2} \nabla_{x}^{2} \hat{f}_{s}=|\omega| \hat{f}_{s}+i \operatorname{sgn} \omega\left(\boldsymbol{h} \cdot \hat{\boldsymbol{f}}_{t}\right), \\
\frac{D}{2} \nabla_{x}^{2} \hat{\boldsymbol{f}}_{t}=|\omega| \hat{\boldsymbol{f}}_{t}+i \operatorname{sgn} \omega\left(\boldsymbol{h} \hat{f}_{s}\right) .
\end{gathered}
$$

The solution of this system can be obtained in the compact form in the realistic limit $\xi_{F} \ll d_{F} \ll \xi_{N}$. Further, we are only interested in the LRT component of the anomalous Green's function which is approximately constant in the ferromagnet for the case under consideration. Up to the first order in the parameter $\tilde{\alpha}\left(p_{s} \xi_{N}\right)\left(\xi_{N} / d_{F}\right)$, which is assumed to be small, we obtain the LRT component in the ferromagnet [104]

$$
\hat{\boldsymbol{f}}_{t}=-\frac{\gamma \xi_{F}^{2} \tilde{\alpha}}{d_{F}|\omega|} \hat{\tau}_{3} \hat{F}_{b c s} \boldsymbol{h} \times\left(\boldsymbol{n} \times \boldsymbol{p}_{s}\right) .
$$

Now we are ready to calculate the enhancement of the DOS in the ferromagnet due to the odd-frequency LRT $[14,50,109]$ $\delta N(\varepsilon)=\frac{1}{2}\left|\hat{\boldsymbol{f}}_{t}^{2}\right|(\omega=i \varepsilon+\delta)$ where $\delta$ is the Dynes parameter. For $d_{F} \gg \xi_{F}$ the contribution to this correction resulting from the short-range triplets already vanishes and, therefore, $\delta N=$ 0 at $B=0$. However, at $B \neq 0$ the LRTs expressed by Eq. (7) start to appear as $\delta N \propto B^{2}$.

Next let us consider the long-range Josephson effect induced by a magnetic field in the setup shown in the inset in Fig. 3. We assume similar conditions on the thickness of $F$ layer $d_{F}$ as above. Having in hand the solutions (7) for left and right $\mathrm{S} / \mathrm{F}$ boundaries we calculate [104] the Josephson current-phase relation in the form $I=I_{c} \sin \phi$ with the critical 


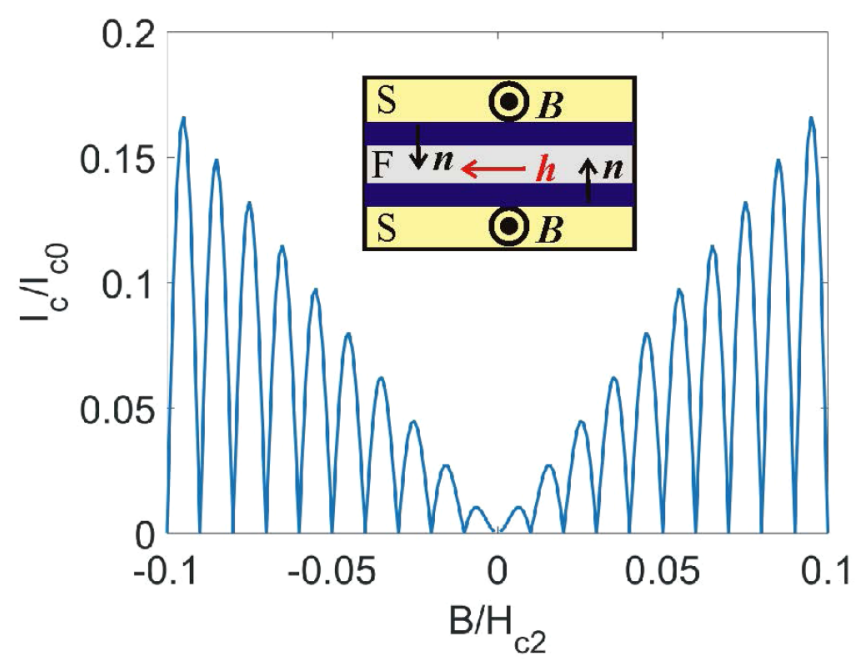

FIG. 3. Interference patterns of the critical current $I_{c}(\Phi)$ for the magnetic-field-induced Josephson effect through the magnetic and SOC interlayers as shown in the inset. We use the parameters $T=$ $0.1 \Delta, d_{F}=\xi$, and $L \lambda_{L}=20 \xi^{2}$ and denote $H_{c 2}=\Phi_{0} / \xi^{2}$.

current [104]

$$
I_{c} / I_{c 0}=\xi^{2}\left(\boldsymbol{p}_{s} \boldsymbol{n}_{h}\right)^{2} \operatorname{Re}\left[\psi\left(\frac{1+i a}{2}\right)-\psi\left(\frac{1}{2}\right)\right],
$$

where $\boldsymbol{n}_{h}=\boldsymbol{h} / h, \psi$ is the digamma function, $a=\Delta / \pi T$, $I_{c 0}=4 \sigma_{F} \Delta\left(\gamma \tilde{\alpha} \xi_{F}\right)^{2} / e d_{F}$, and $\sigma_{F}$ is the ferromagnet conductivity. The physical origin of unusual behavior in Fig. 3 is determined by the mechanism which is the main topic of this Rapid Communication, namely, the generation of long-range spin-triplet correlations by an external magnetic field. As we have obtained in Eq. (7), the amplitude of long-range spin triplets is proportional to the condensate momentum $\boldsymbol{p}_{s}$ which is generated by the external magnetic field through the Meissner effect. In the SFS junction with tunnel interface barriers and weak proximity effect [110] $p_{s}\left(d_{F} / 2\right)=-p_{s}\left(-d_{F} / 2\right)=$ $B \lambda_{L} / \Phi_{0}$. Hence the amplitude of critical current grows as $I_{c} \propto B^{2}$ for a small external magnetic field, when the total flux through the junction area $\Phi=2 \lambda_{L} L B$ is small, $\Phi \ll \Phi_{0}$. Here $L$ is the length of the junction. For larger fields we need to take into account phase variation along the junction which leads to the usual factor $(L \sin \phi) / \phi$ in the critical current where $\phi=$ $2 \pi \Phi / \Phi_{0}$. As a result, we obtain $I_{c} \propto B$ envelope dependence of the critical current shown in Fig. 3. This growth is bounded from above by the depairing effects from which the most important one is expected to be the vortex entry [111] to the superconducting leads caused by the external magnetic field $B$. This happens at fields $H_{v}$ when the condensate momentum at the edge becomes of the order of the critical depairing value so that $p_{s} \xi \sim 1$. Depending on the level of disorder, one can have the values [111] $H_{v} / H_{c 2} \sim 0.1-0.19$. For such samples the orbital effects can be neglected at fields $B<0.1 H_{c 2}$ as in Fig. 3.

The critical current magnitude can be estimated using Eq. (8) with the typical junction parameters [49]. Assuming that the junction area is $50 \times 50 \mu \mathrm{m}^{2}, \sigma_{F} \sim(50 \mu \Omega \mathrm{cm})^{-1}$, $d_{F} \sim \gamma^{-1} \sim 5 \xi_{F}$, and $D \sim 10 \mathrm{~cm}^{2} / \mathrm{s}, h \sim 500 \mathrm{~K}$ so that $\xi_{F} \sim$ $3 \mathrm{~nm}$. For the superconducting gap in $\mathrm{Nb} \Delta \sim 10 \mathrm{~K}$ so that the critical current is $I_{c} \sim 10^{-1}\left(p_{s} \xi\right)^{2} \tilde{\alpha}^{2}$ A. Taking $\tilde{\alpha} \sim$ $0.1-1[27,89,112]$ and $p_{s} \xi=0.3$, we get the current $I_{c} \sim$ $10^{-2}-10^{-4}$ A which is much larger than in SFS junctions where LRTs are generated by the magnetic inhomogeneity [49]. This $I_{c}$ is much larger than obtained for the planar geometry [98] because in our mechanism there are no physical limitations on the junction cross section.

The $I_{c}(B)$ pattern in Fig. 3 drastically differs from the ones observed previously in nonferromagnetic Josephson junctions with SOC [113,114] and ferromagnetic ones without SOC [115]. This behavior can be considered as the fingerprint of LRTs produced due to the presence of interfacial Rashba SOC. The direction of $\boldsymbol{h}$ in Fig. 3 can be pinned tailoring the F layer shape anisotropy so that the easy axis is perpendicular to $\boldsymbol{B}$. Such scenarios were realized experimentally and coercive fields of about $0.3 \mathrm{~T}$ for $\mathrm{Fe}$ samples were measured [116] which exceeds the field range in Fig. 3 provided that $H_{c 2}<3 \mathrm{~T}$, which is typical for $\mathrm{Nb}$ superconducting leads.

In conclusion, we have demonstrated a general mechanism which provides the generation of odd-frequency spin-triplet superconductivity by moving the superconducting condensate in homogeneous ferromagnets. Physically the effect originates from a coupling of a Cooper-pair spin with its orbital motion via SOC. The effect leads to the appearance of LRT correlations in experimentally relevant $\mathrm{S} / \mathrm{F}$ hybrids and long-range Josephson effect switchable by an externally applied magnetic field.

The work of M.A.S. was supported by the Academy of Finland (Project No. 297439) and Russian Science Foundation (Grant No. 19-19-00594). The work of I.V.B. and A.M.B. has been carried out within the state task of ISSP RAS with support from RFBR Grants No.19-02-00466, 18-52-45011 and 18-02-00318. I.V.B. also acknowledges the financial support by Foundation for the Advancement of Theoretical Physics and Mathematics "BASIS."
[1] M. Tinkham, Introduction to Superconductivity (Courier Corporation, Chelmsford, MA, 1996).

[2] V. Berezinskii, JETP Lett. 20, 287 (1974).

[3] D. Belitz and T. R. Kirkpatrick, Phys. Rev. B 46, 8393 (1992).

[4] D. Belitz and T. R. Kirkpatrick, Phys. Rev. B 60, 3485 (1999).

[5] A. Balatsky and E. Abrahams, Phys. Rev. B 45, 13125 (1992).
[6] E. Abrahams, A. Balatsky, D. J. Scalapino, and J. R. Schrieffer, Phys. Rev. B 52, 1271 (1995).

[7] P. Coleman, E. Miranda, and A. Tsvelik, Phys. Rev. B 49, 8955 (1994).

[8] A. F. Volkov, F. S. Bergeret, and K. B. Efetov, Phys. Rev. Lett. 90, 117006 (2003).

[9] F. S. Bergeret, A. F. Volkov, and K. B. Efetov, Rev. Mod. Phys. 77, 1321 (2005). 
[10] Y. V. Fominov, Y. Tanaka, Y. Asano, and M. Eschrig, Phys. Rev. B 91, 144514 (2015).

[11] J. Linder and A. V. Balatsky, Rev. Mod. Phys. 91, 045005 (2019).

[12] A. M. Black-Schaffer and A. V. Balatsky, Phys. Rev. B 86, 144506 (2012).

[13] A. M. Black-Schaffer and A. V. Balatsky, Phys. Rev. B 88, 104514 (2013).

[14] A. Di Bernardo, S. Diesch, Y. Gu, J. Linder, G. Divitini, C. Ducati, E. Scheer, M. G. Blamire, and J. W. A. Robinson, Nat. Commun. 6, 8053 (2015).

[15] L. Komendová and A. M. Black-Schaffer, Phys. Rev. Lett. 119, 087001 (2017).

[16] J. Cayao and A. M. Black-Schaffer, Phys. Rev. B 96, 155426 (2017).

[17] J. Cayao, C. Triola, and A. M. Black-Schaffer, Eur. Phys. J.: Spec. Top. 229, 545 (2020).

[18] C. Triola and A. M. Black-Schaffer, Phys. Rev. B 97, 064505 (2018).

[19] J. Cayao and A. M. Black-Schaffer, Phys. Rev. B 98, 075425 (2018).

[20] P. Dutta and A. M. Black-Schaffer, Phys. Rev. B 100, 104511 (2019).

[21] P. O. Sukhachov, V. Juričić, and A. V. Balatsky, Phys. Rev. B 100, 180502(R) (2019).

[22] M. Alidoust, A. Zyuzin, and K. Halterman, Phys. Rev. B 95, 045115 (2017).

[23] Y. Tanaka, Y. Tanuma, and A. A. Golubov, Phys. Rev. B 76, 054522 (2007).

[24] Y. Asano, Y. Sawa, Y. Tanaka, and A. A. Golubov, Phys. Rev. B 76, 224525 (2007).

[25] Y. Asano and Y. Tanaka, Phys. Rev. B 87, 104513 (2013).

[26] T. Yokoyama, Y. Tanaka, and A. A. Golubov, Phys. Rev. B 78, 012508 (2008).

[27] N. Banerjee, J. A. Ouassou, Y. Zhu, N. A. Stelmashenko, J. Linder, and M. G. Blamire, Phys. Rev. B 97, 184521 (2018).

[28] N. Satchell and N. O. Birge, Phys. Rev. B 97, 214509 (2018).

[29] N. Satchell, R. Loloee, and N. O. Birge, Phys. Rev. B 99, 174519 (2019).

[30] K.-R. Jeon, X. Montiel, C. Ciccarelli, H. Kurebayashi, L. F. Cohen, S. Komori, M. G. Blamire, and J. W. Robinson, Phys. Rev. X 10, 031020 (2020).

[31] K.-R. Jeon, C. Ciccarelli, H. Kurebayashi, L. F. Cohen, S. Komori, J. W. A. Robinson, and M. G. Blamire, Phys. Rev. B 99, 144503 (2019).

[32] K.-R. Jeon, C. Ciccarelli, H. Kurebayashi, L. F. Cohen, X. Montiel, M. Eschrig, S. Komori, J. W. A. Robinson, and M. G. Blamire, Phys. Rev. B 99, 024507 (2019).

[33] K.-R. Jeon, C. Ciccarelli, H. Kurebayashi, L. F. Cohen, X. Montiel, M. Eschrig, T. Wagner, S. Komori, A. Srivastava, J. W. A. Robinson, and M. G. Blamire, Phys. Rev. Appl. 11, 014061 (2019).

[34] K.-R. Jeon, C. Ciccarelli, A. J. Ferguson, H. Kurebayashi, L. F. Cohen, X. Montiel, M. Eschrig, J. W. A. Robinson, and M. G. Blamire, Nat. Mater. 17, 499 (2018).

[35] S. Oh, D. Youm, and M. Beasley, Appl. Phys. Lett. 71, 2376 (1997).

[36] L. R. Tagirov, Phys. Rev. Lett. 83, 2058 (1999).
[37] A. Kadigrobov, R. Shekhter, and M. Jonson, Europhys. Lett. 54, 394 (2001).

[38] J. Y Gu, C.-Y. You, J. S. Jiang, J. Pearson, Y. B. Bazaliy, and S. D. Bader, Phys. Rev. Lett. 89, 267001 (2002).

[39] Y. V. Fominov, A. A. Golubov, and M. Y. Kupriyanov, J. Exp. Theor. Phys. Lett. 77, 510 (2003).

[40] Y. V. Fominov, A. A. Golubov, T. Y. Karminskaya, M. Y. Kupriyanov, R. Deminov, and L. R. Tagirov, JETP Lett. 91, 308 (2010).

[41] K. Halterman, P. H. Barsic, and O. T. Valls, Phys. Rev. Lett. 99, 127002 (2007).

[42] K. Halterman, O. T. Valls, and P. H. Barsic, Phys. Rev. B 77, 174511 (2008).

[43] J. Zhu, I. N. Krivorotov, K. Halterman, and O. T. Valls, Phys. Rev. Lett. 105, 207002 (2010).

[44] M. Alidoust, K. Halterman, and J. Linder, Phys. Rev. B 89, 054508 (2014).

[45] F. S. Bergeret, A. F. Volkov, and K. B. Efetov, Phys. Rev. Lett. 86, 4096 (2001).

[46] F. S. Bergeret, A. F. Volkov, and K. B. Efetov, Phys. Rev. Lett. 86, 3140 (2001).

[47] M. Houzet and A. I. Buzdin, Phys. Rev. B 76, 060504(R) (2007).

[48] R. Keizer, S. Goennenwein, T. Klapwijk, G. Miao, G. Xiao, and A. Gupta, Nature (London) 439, 825 (2006).

[49] Y. V. Fominov, A. F. Volkov, and K. B. Efetov, Phys. Rev. B 75, 104509 (2007).

[50] V. Braude and Y. V. Nazarov, Phys. Rev. Lett. 98, 077003 (2007).

[51] M. Eschrig and T. Löfwander, Nat. Phys. 4, 138 (2008).

[52] M. Eschrig, J. Kopu, J. C. Cuevas, and G. Schön, Phys. Rev. Lett. 90, 137003 (2003).

[53] J. W. A. Robinson, G. B. Halász, A. I. Buzdin, and M. G. Blamire, Phys. Rev. Lett. 104, 207001 (2010).

[54] J. W. A. Robinson, J. D. S. Witt, and M. G. Blamire, Science 329, 59 (2010).

[55] A. Singh, C. Jansen, K. Lahabi, and J. Aarts, Phys. Rev. X 6, 041012 (2016).

[56] T. S. Khaire, M. A. Khasawneh, W. P. Pratt, Jr., and N. O. Birge, Phys. Rev. Lett. 104, 137002 (2010).

[57] S. Mironov and A. Buzdin, Phys. Rev. B 92, 184506 (2015).

[58] K. Halterman and M. Alidoust, Phys. Rev. B 94, 064503 (2016).

[59] M. Alidoust and K. Halterman, Phys. Rev. B 97, 064517 (2018).

[60] A. Srivastava, L. A. B. Olde Olthof, A. Di Bernardo, S. Komori, M. Amado, C. Palomares-Garcia, M. Alidoust, K. Halterman, M. G. Blamire, and J. W. A. Robinson, Phys. Rev. Appl. 8, 044008 (2017).

[61] J. Linder and J. W. A. Robinson, Nat. Phys. 11, 307 (2015).

[62] M. Eschrig, Rep. Prog. Phys. 78, 104501 (2015).

[63] Z. Niu, Appl. Phys. Lett. 101, 062601 (2012).

[64] S. B. Chung, H.-J. Zhang, X.-L. Qi, and S.-C. Zhang, Phys. Rev. B 84, 060510(R) (2011).

[65] O. Dimitrova and M. V. Feigel'man, Phys. Rev. B 76, 014522 (2007).

[66] A. G. Mal'shukov, S. Sadjina, and A. Brataas, Phys. Rev. B 81, 060502(R) (2010). 
[67] I. V. Bobkova and A. M. Bobkov, Phys. Rev. B 95, 184518 (2017).

[68] G. Tkachov, Phys. Rev. Lett. 118, 016802 (2017).

[69] S. Mironov and A. Buzdin, Phys. Rev. Lett. 118, 077001 (2017).

[70] M. Alidoust and K. Halterman, J. Phys.: Condens. Matter 27, 235301 (2015).

[71] M. Alidoust and K. Halterman, New J. Phys. 17, 033001 (2015).

[72] S.-i. Hikino, J. Phys. Soc. Jpn. 87, 074707 (2018).

[73] F. Konschelle, I. V. Tokatly, and F. S. Bergeret, Phys. Rev. B 92, 125443 (2015).

[74] F. S. Bergeret and I. V. Tokatly, Phys. Rev. Lett. 110, 117003 (2013).

[75] F. S. Bergeret and I. V. Tokatly, Phys. Rev. B 89, 134517 (2014).

[76] I. V. Tokatly, Phys. Rev. B 96, 060502(R) (2017).

[77] I. V. Bobkova and Y. S. Barash, J. Exp. Theor. Phys. Lett. 80, 494 (2004).

[78] F. S. Bergeret and I. V. Tokatly, Europhys. Lett. 110, 57005 (2015)

[79] G. Campagnano, P. Lucignano, D. Giuliano, and A. Tagliacozzo, J. Phys.: Condens. Matter 27, 205301 (2015).

[80] S. S. Pershoguba, K. Bjornson, A. M. Black-Schaffer, and A. V. Balatsky, Phys. Rev. Lett. 115, 116602 (2015).

[81] A. G. Mal'shukov, Phys. Rev. B 93, 054511 (2016).

[82] A. G. Mal'shukov, Phys. Rev. B 98, 054504 (2018).

[83] A. Buzdin, Phys. Rev. Lett. 101, 107005 (2008).

[84] F. Konschelle and A. Buzdin, Phys. Rev. Lett. 102, 017001 (2009).

[85] J. W. A. Robinson, A. V. Samokhvalov, and A. I. Buzdin, Phys. Rev. B 99, 180501(R) (2019).

[86] X. Montiel and M. Eschrig, Phys. Rev. B 98, 104513 (2018).

[87] E. I. Rashba and V. I. Sheka, Fiz. Tverd. Tela 2, 162 (1959).

[88] G. Bihlmayer, O. Rader, and R. Winkler, New J. Phys. 17, 050202 (2015).

[89] C. R. Ast, J. Henk, A. Ernst, L. Moreschini, M. C. Falub, D. Pacilé, P. Bruno, K. Kern, and M. Grioni, Phys. Rev. Lett. 98, 186807 (2007).

[90] G. Bihlmayer, Y. M. Koroteev, P. Echenique, E. Chulkov, and S. Blügel, Surf. Sci. 600, 3888 (2006).

[91] A. Manchon, H. C. Koo, J. Nitta, S. Frolov, and R. Duine, Nat. Mater. 14, 871 (2015).

[92] L. P. Gor'kov and E. I. Rashba, Phys. Rev. Lett. 87, 037004 (2001).

[93] V. M. Edelstein, Phys. Rev. B 67, 020505(R) (2003).

[94] C. R. Reeg and D. L. Maslov, Phys. Rev. B 92, 134512 (2015).
[95] F. Konschelle, I. V. Tokatly, and F. S. Bergeret, Phys. Rev. B 94, 014515 (2016).

[96] A. Kohen, T. Proslier, T. Cren, Y. Noat, W. Sacks, H. Berger, and D. Roditchev, Phys. Rev. Lett. 97, 027001 (2006).

[97] S. H. Jacobsen, J. A. Ouassou, and J. Linder, Phys. Rev. B 92, 024510 (2015).

[98] J. R. Eskilt, M. Amundsen, N. Banerjee, and J. Linder, Phys. Rev. B 100, 224519 (2019).

[99] B. Bujnowski, R. Biele, and F. S. Bergeret, Phys. Rev. B 100, 224518 (2019).

[100] A. J. Leggett, Rev. Mod. Phys. 47, 331 (1975).

[101] W. V. Budzinski, M. P. Garfunkel, and R. W. Markley, Phys. Rev. B 7, 1001 (1973).

[102] V. Edelstein, Solid State Commun. 73, 233 (1990).

[103] G. Eilenberger, Z. Phys. 214, 195 (1968).

[104] See Supplemental Material at http://link.aps.org/ supplemental/10.1103/PhysRevB.102.100507 for a discussion of the details of the calculation.

[105] A. Singh, S. Voltan, K. Lahabi, and J. Aarts, Phys. Rev. X 5, 021019 (2015).

[106] K. Lahabi, M. Amundsen, J. A. Ouassou, E. Beukers, M. Pleijster, J. Linder, P. Alkemade, and J. Aarts, Nat. Commun. 8, 2056 (2017).

[107] A. Iovan, T. Golod, and V. M. Krasnov, Phys. Rev. B 90, 134514 (2014).

[108] M. Kupriyanov and V. Lukichev, Sov. Phys. JETP 67, 1163 (1988).

[109] T. Yokoyama, Y. Tanaka, and A. A. Golubov, Phys. Rev. B 72, 052512 (2005).

[110] A. V. Galaktionov and A. D. Zaikin, Phys. Rev. B 67, 184518 (2003).

[111] R. W. De Blois and W. De Sorbo, Phys. Rev. Lett. 12, 499 (1964).

[112] S.-T. Lo, S.-W. Lin, Y.-T. Wang, S.-D. Lin, and C.-T. Liang, Sci. Rep. 4, 5438 (2014).

[113] H. J. Suominen, J. Danon, M. Kjaergaard, K. Flensberg, J. Shabani, C. J. Palmstrøm, F. Nichele, and C. M. Marcus, Phys. Rev. B 95, 035307 (2017).

[114] A. Assouline, C. Feuillet-Palma, N. Bergeal, T. Zhang, A. Mottaghizadeh, A. Zimmers, E. Lhuillier, M. Eddrie, P. Atkinson, M. Aprili et al., Nat. Commun. 10, 1 (2019).

[115] M. Kemmler, M. Weides, M. Weiler, M. Opel, S. T. B. Goennenwein, A. S. Vasenko, A. A. Golubov, H. Kohlstedt, D. Koelle, R. Kleiner, and E. Goldobin, Phys. Rev. B 81, 054522 (2010).

[116] T. E. Golikova, F. Hübler, D. Beckmann, I. E. Batov, T. Y. Karminskaya, M. Y. Kupriyanov, A. A. Golubov, and V. V. Ryazanov, Phys. Rev. B 86, 064416 (2012). 